\title{
Sangrado tardío por déficit de factor XIII tras citorreducción y quimioterapia intraoperatoria hipertérmica
}

\author{
Inmaculada Hernández ${ }^{1}$, Laura Soriano ${ }^{1}$, Estrella Terradillos ${ }^{1}$, Patricia Piñeiro ${ }^{1}$, Wenceslao Vasquez ${ }^{2}$ \\ y Patricia Duque ${ }^{1 *}$ \\ ${ }^{1}$ Anestesiología y Reanimación; ${ }^{2}$ Cirugía General. Hospital General Universitario Gregorio Marañón, Madrid, España
}

\begin{abstract}
Resumen
Presentamos el caso de una paciente que se somete a una citorreducción y quimioterapia intraoperatoria hipertérmica con oxaliplatino por carcinomatosis peritoneal secundaria a carcinoma apendicular. Presenta una evolución tórpida secundaria a sangrado intraabdominal tardío postoperatorio, que requiere tratamiento hemoterápico intensivo. La paciente se monitoriza mediante tromboelastometría. Tras estudio de hemostasia, es diagnosticada de hiperfibrinólisis asociada a déficit de factor XIII y trombopenia por consumo. Realizamos una revisión de la literatura reciente en relación con la alteración de la coagulación y la quimioterapia hipertérmica con oxaliplatino.
\end{abstract}

Palabras clave: FXIII. Quimioterapia intraoperatoria hipertérmica. Sangrado.

\section{Late bleeding due to FXIII deficiency following cytoreductive surgery and hyperthermic intraperitoneal chemotherapy}

\begin{abstract}
We present the case of a patient undergoing cytoreductive surgery and hyperthermic intraperitoneal chemotherapy with oxaliplatin for peritoneal carcinomatosis secondary to appendicular carcinoma. She developed late postoperative intra-abdominal bleeding requiring intensive blood transfusion. She was monitored with thromboelastometry and diagnosed of hyperfibrinolysis associated with FXIII deficiency and thrombocytopenia. We conduct a literature review about coagulopathy after cytoreductive surgery and hyperthermic intraperitoneal chemotherapy.
\end{abstract}

Key words: FXIII. Hyperthermic intraperitoneal chemotherapy. Bleeding.

Correspondencia:

Patricia Duque

E-mail: patriduque@gmail.com
Disponible en internet: 05-02-2019

Fecha de recepción: 30-06-2018

Fecha de aceptación: 29-09-2018

DOI: 10.24875/RAA. 18000017
Rev Argent Anestesiol. 2018;76(2):93-98

www.revistaargentinaanestesiologia.com

0370-7792 @ 2018 Federación Argentina de Asociaciones, Anestesia, Analgesia y Reanimación. Publicado por Permanyer México SA de CV. Este es un artículo Open Access bajo la licencia CC BY-NC-ND (http://creativecommons.org/licenses/by-nc-nd/4.0/). 
Tabla 1. Evolución analítica durante la estancia en la Unidad de Cuidados Intensivos

\begin{tabular}{|c|c|c|c|c|c|c|c|}
\hline & $\begin{array}{l}\text { Primer } \\
\text { día de } \\
\text { ingreso }\end{array}$ & $\begin{array}{l}\text { Tercer } \\
\text { día de } \\
\text { ingreso }\end{array}$ & $\begin{array}{l}\text { Cuarto día: } \\
\text { cirugía } \\
\text { urgente }\end{array}$ & $\begin{array}{l}\text { Octavo día: segunda } \\
\text { reintervención } \\
\text { (Fig. 1) }\end{array}$ & $\begin{array}{l}\text { Octavo } \\
\text { día } \\
\text { (Fig. 2) }\end{array}$ & $\begin{array}{l}\text { Noveno día } \\
\text { postoperatorio } \\
\text { (Fig. 3) }\end{array}$ & $\begin{array}{c}\text { Alta } \\
\text { de } \\
\text { UCIO }\end{array}$ \\
\hline $\mathrm{Hb}(\mathrm{mg} / \mathrm{dl})$ & 10.3 & 7.3 & 8.8 & 7.3 & 10.9 & 8.2 & 9.4 \\
\hline Plaquetas $\left(10^{3} / \mathrm{mcl}\right)$ & 141 & 50 & 46 & 77 & 61 & 41 & 87 \\
\hline $\begin{array}{l}\text { Recuento manual de } \\
\text { plaquetas }\left(10^{3} / \mathrm{mcl}\right)\end{array}$ & & & & & & 51 & \\
\hline Leucocitos $\left(10^{3} / \mathrm{mcl}\right)$ & 11.5 & 7.3 & 8.8 & 6.1 & 3.9 & 2.3 & 15 \\
\hline $\begin{array}{l}\text { Dosificación funcional } \\
\text { de factores }\end{array}$ & & & & & & $\begin{array}{l}\text { Factor II: C } 102 \% \\
\text { Factor V: C } 131 \% \\
\text { Factor VII: C } 96 \% \\
\text { Factor VIII: C } 350 \% \\
\text { Factor IX: C } 224 \% \\
\text { Factor X: C } 89.9 \% \\
\text { Factor XI: C } 112 \% \\
\text { Factor XII: C 56\% } \\
\text { Factor XIII: Ag } 24 \%\end{array}$ & \\
\hline $\mathrm{TP}(\mathrm{s})$ & 13.6 & 12.5 & 13.6 & 13.1 & 11.7 & 12.5 & 11.7 \\
\hline INR & 1.13 & 1.05 & 1.13 & 1.09 & 0.98 & 1.05 & 0.98 \\
\hline APTT (s) & 28.7 & 30.8 & 28.8 & 26.5 & 29.1 & 29.3 & 24.1 \\
\hline Ratio APTT & 0.96 & 1.03 & 0.96 & 0.88 & 0.97 & 0.98 & 0.8 \\
\hline Fibrinógeno (mg/dL) & 473 & 404 & 233 & 353 & 573 & 695 & 502 \\
\hline
\end{tabular}

APTT: tiempo de tromboplastina parcial activada; Hb: Hemoglobina; INR: relación normalizada internacional; TP: tiempo de protrombina.

\section{Caso clínico}

Se trata de una paciente mujer de 55 años, sin antecedentes médico-quirúrgicos de interés ni historia de sangrados previos, que ingresa en la Unidad de Cuidados Intensivos Quirúrgicos (UCIQ) tras citorreducción peritoneal (omentectomía mayor y menor, peritonectomías diafragmática derecha y pericárdica, pelviperitonectomía con histerectomía y doble anexectomía) y quimioterapia intraoperatoria hipertérmica $(\mathrm{QIOH})$ con oxaliplatino, según régimen habitual (oxaliplatino $450 \mathrm{mg} / \mathrm{m}^{2}$ de superficie corporal, intraperitoneal, y 5 -fluorouracilo $400 \mathrm{mg} / \mathrm{m}^{2}$ de superficie corporal, intravenoso), por carcinomatosis peritoneal secundaria a carcinoma apendicular. Durante la cirugía la paciente se mantiene estable sin precisar soporte inotrópico ni transfusión de hemoderivados. La reposición de fluidos se realiza guiada por termodilución transpulmonar, con administración de $3,750 \mathrm{ml}$ de cristaloides y $600 \mathrm{ml}$ de coloides en 10 horas de cirugía. Se extuba al finalizar la cirugía, sin incidencias. La paciente ingresa ventilatoria y hemodinámicamente estable, con buen control analgésico por catéter epidural torácico y analítica sin alteraciones significativas (hemoglobina $10.3 \mathrm{~g} / \mathrm{dl}$, fibrinógeno $323 \mathrm{mg} / \mathrm{dl}$ y plaquetas $172 \times 10^{3} / \mathrm{mcl}$ ).
A partir del segundo día postoperatorio presenta una evolución tórpida caracterizada, desde el punto de vista clínico, por episodios de descompensación hemodinámica en relación con un shock hemorrágico con sangrado por los drenajes y puntos de inserción y, desde el punto de vista analítico, por anemización y trombopenia progresivas a pesar de la transfusión de concentrados de hematíes y plaquetas, que obligan a dos reintervenciones por shock hemorrágico. Se realiza frotis de sangre periférica y se confirma la trombopenia evidenciada en la analítica, sugestiva de ser secundaria a consumo por sangrado. En la tabla 1 se puede observar la evolución analítica.

Con respecto a los requerimientos transfusionales, durante el tercer día de ingreso se transfunden dos concentrados de hematíes. El cuarto día de ingreso, justo antes de la primera reintervención, se transfunden tres concentrados de hematíes y un pool de plaquetas. Entre los días cuarto y séptimo de ingreso es necesaria la transfusión de dos concentrados de hematíes y un pool de plaquetas al día, mientras que el octavo día postoperatorio (segunda reintervención) es preciso transfundir cuatro concentrados de hematíes y dos pools de plaquetas. 


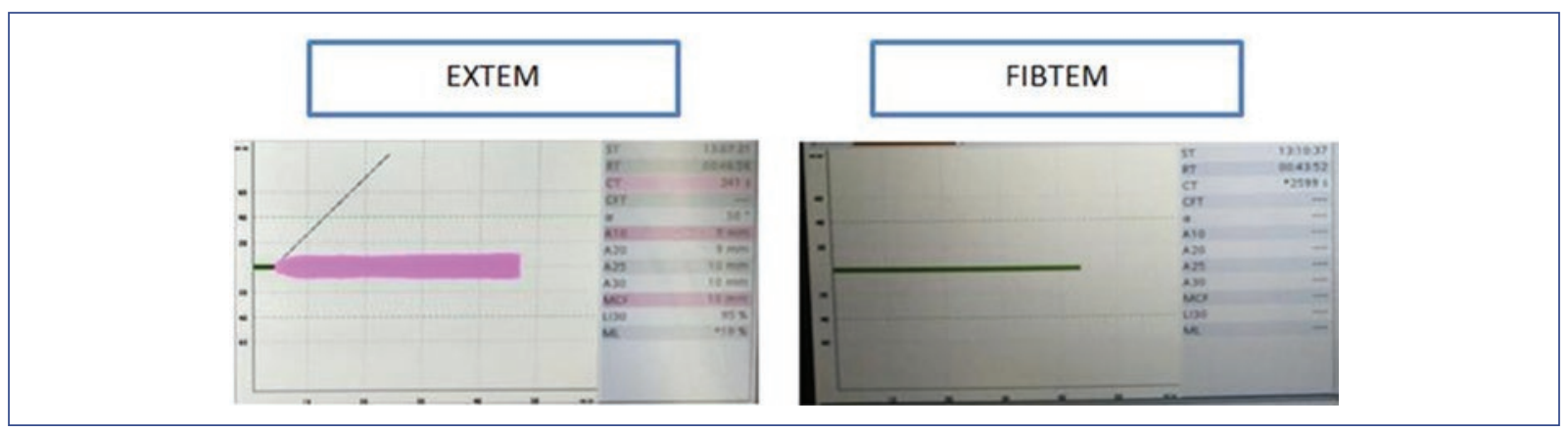

Figura 1. Primera tromboelastometría (paciente inestable con sangrado activo). Se observa un EXTEM con déficit global de factores, evidenciado por un tiempo de coagulación (CT) muy alargado (CT = 241 s) y una amplitud máxima del coágulo muy disminuida (amplitud máxima de coágulo $(M C F)=10 \mathrm{~mm}$ ). El coágulo en el FIBTEM no llega ni siquiera a formarse (línea plana), lo que indica depleción de fibrinógeno probablemente en relación con hiperfibrinólisis.

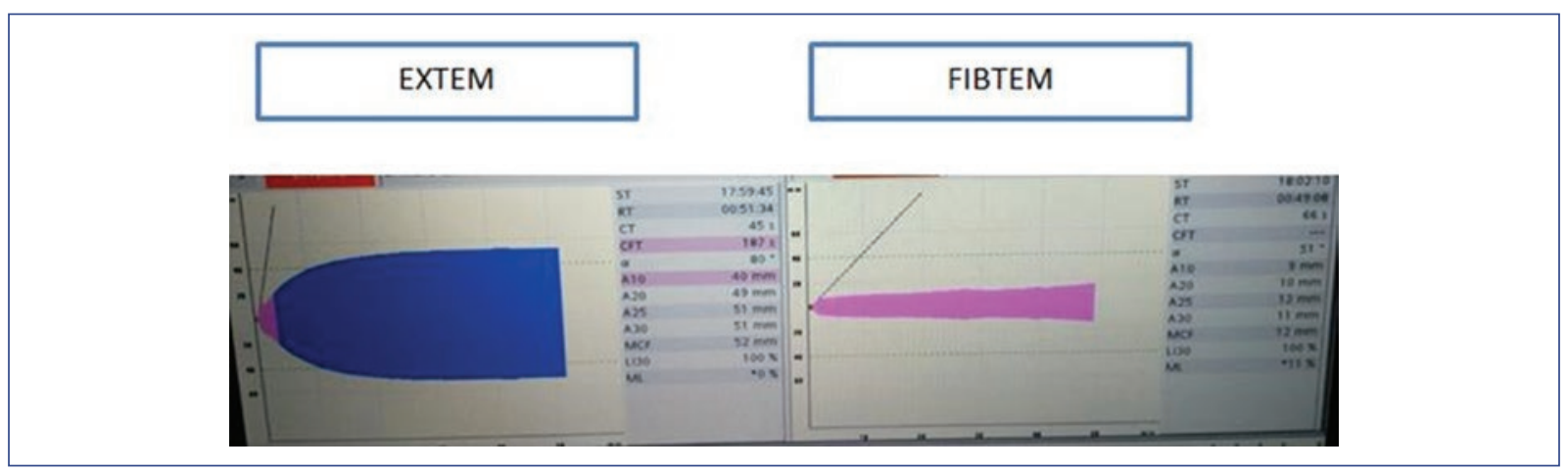

Figura 2. Segunda tromboelastometría (paciente estable sin sangrado). EXTEM y FIBTEM dentro de los límites normales. Indica que nuestra paciente está formando un coágulo lo suficientemente estable como para impedir el sangrado.

En relación con la evolución clínica, en el cuarto día postoperatorio se somete a una reintervención urgente, donde se objetiva hematoma de pared en el tercio inferior de la laparotomía y hematomas perihepático, periesplénico y pélvico, con hemoperitoneo de 2 litros. Se realiza lavado abundante, drenaje de hematomas, hemostasia y cierre. La paciente se recupera clínicamente hasta el cuarto día tras la reintervención quirúrgica (octavo día postoperatorio), cuando presenta un nuevo shock hemorrágico secundario a sangrado en la región perihepática anterior, pelvis, raíz del mesenterio y pared abdominal inferior izquierda. Se indica nueva reintervención urgente para evacuar los hematomas, realizar packing abdominal y laparostomía para cierre diferido.

Tras esta segunda reintervención, la paciente continúa presentando sangrado tanto por los drenajes abdominales como por laparostomía y clínicamente está inestable y precisa perfusión continua de noradrenalina a $0.5 \mathrm{mcg} / \mathrm{kg} / \mathrm{min}$ para mantener la tensión arterial media $($ TAM) $>60 \mathrm{mmHg}$. Sorprendentemente, las pruebas convencionales de coagulación eran rigurosamente normales y presentaba anemia y trombopenia (Tabla 1). Ante estos hallazgos, decidimos realizar control evolutivo por tromboelastometría (Fig. 1). Se objetiva unos valores en tromboelastometría de vía extrínseca (EXTEM) y tromboelastometría de vía intrínseca (FIBTEM) sugestivos de hiperfibrinólisis, consumo de fibrinógeno, plaquetas y factores de la coagulación. Dados los resultados de la tromboelastometría y el hemograma, se transfunden $1 \mathrm{~g}$ de ácido tranexámico más perfusión continua a $10 \mathrm{mg} / \mathrm{kg} / \mathrm{h}$, dos concentrados de hematíes, dos pools de plaquetas, $6 \mathrm{~g}$ de fibrinógeno y 1,000 $\mathrm{U}$ de complejo protrombínico activado. Esto permite la retirada del soporte vasoactivo y la reducción del sangrado por los drenajes hasta hacerse mínimo en las siguientes horas. En la tromboelastometría de control (6 horas después de la anterior), se objetiva una corrección casi completa (Fig. 2). Suspendemos la perfusión continua de ácido tranexámico y la paciente no vuelve a precisar transfusión de hemoderivados. 


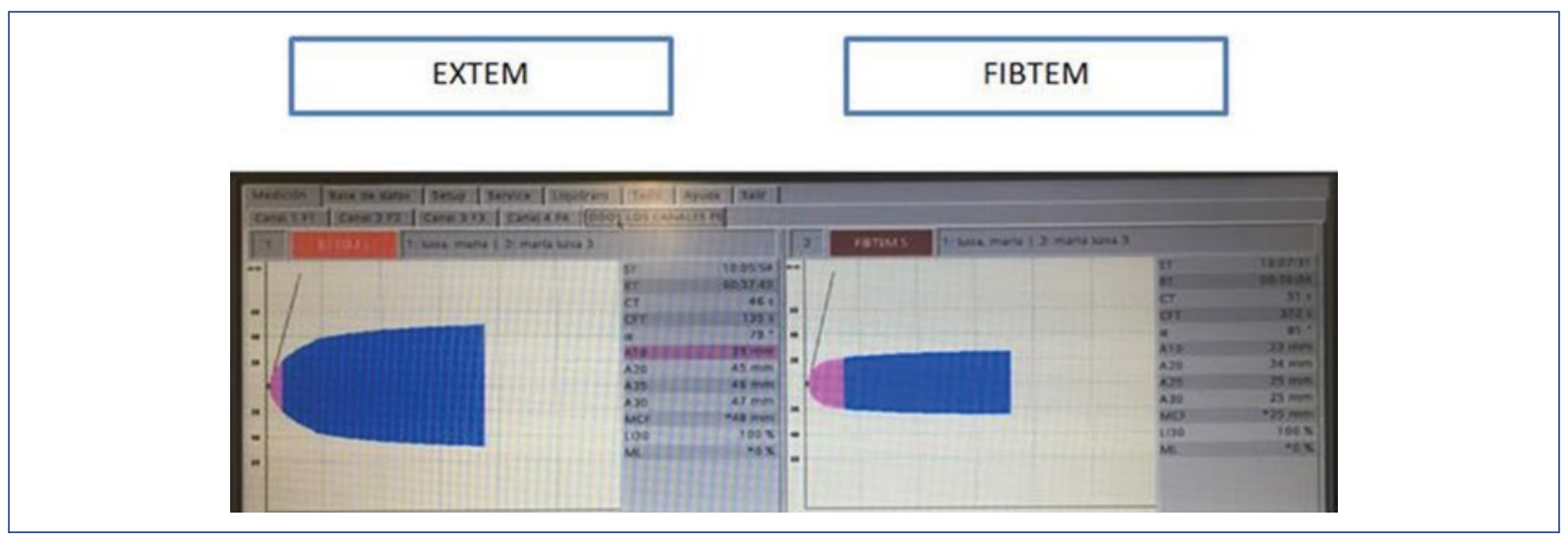

Figura 3. Tercera tromboelastometría (paciente estable, sin sangrado, previo a cierre de pared). En el EXTEM se objetiva una amplitud máxima del coágulo en el límite bajo de la normalidad (MCF = $48 \mathrm{~mm}$ ), mientras que en el FIBTEM la amplitud del coágulo está claramente aumentada (amplitud máxima de coágulo (MCF) $=25 \mathrm{~mm}$ ). Esto indica probablemente un déficit plaquetario compensado por la acción del fibrinógeno.

Al día siguiente, la paciente se mantiene estable. Se realiza un nuevo control tromboelastométrico donde se objetiva un déficit plaquetario compensado por la acción del fibrinógeno, lo que da como resultado final un coágulo estable (Fig. 3). En este momento se obtiene, además, la dosificación funcional de factores y proteínas anticoagulantes (antitrombina III (ATIII), proteína C y proteína S). La paciente es trasladada a quirófano para revisión y cierre de la pared abdominal (noveno día postoperatorio), que se realizan sin incidencias reseñables ni requerimientos transfusionales. Tras el cierre de la pared abdominal, la paciente presenta buena evolución clínica. En relación con los resultados del estudio de hemostasia se objetivó un déficit grave de factor XIII (FXIII) con una actividad del $24 \%$ (estando el resto de perfil pro y anticoagulante en el rango de la normalidad), que se trató con la administración de plasma fresco congelado: $1 \mathrm{U} / 24 \mathrm{~h}$ durante 2 días. El déficit se corrige, según se evidencia en controles sucesivos: actividad del $95 \%$ el día 16 postoperatorio y del $154 \%$ al alta hospitalaria. En el frotis realizado el día 14 postoperatorio, la cifra de plaquetas ya había aumentado a 112,000/mcl. No tuvo más eventos hemorrágicos. Se trasladó a planta de hospitalización de Cirugía General el día 18 postoperatorio, sin más incidencias. Se retiró el catéter epidural el día 19 postoperatorio sin incidencias. La paciente fue dada de alta a su domicilio a los 35 días de ingreso.

\section{Discusión}

Aproximadamente un tercio de los pacientes que se someten a QIOH presentan alteración de la coagulación con tendencia al sangrado, en probable relación con las grandes pérdidas proteicas y el desplazamiento de fluidos que se genera en esta cirugía, la hipo o hipertermia, el tipo de tumor y la toxicidad del quimioterápico ${ }^{1-3}$. Una revisión retrospectiva reciente ${ }^{4}$ encuentra que un $40 \%$ de pacientes tratados con citorreducción más $\mathrm{QIOH}$ ( $75 \%$ con oxaliplatino) presentaban una coagulación alterada en el postoperatorio, aunque solo un $2 \%$ precisó revisión quirúrgica. Llama la atención que estos autores utilizaron ácido tranexámico en un $60 \%$ de sus pacientes, aunque no explican por qué, ya que en este estudio la técnica anestésica no estaba estandarizada. Un estudio observacional prospectivo español ${ }^{5}$ encuentra que la cirugía de citorreducción más QIOH se acompaña de disfunción del fibrinógeno objetivado por alteraciones tromboelastométricas (disminución de MCF en el test FIBTEM de la tromboelastometría). La administración de ácido tranexámico empírico y fibrinógeno preventivo desde el inicio de la cirugía y durante la misma se ha asociado a una disminución de las pérdidas hemáticas intraoperatorias sin objetivar un aumento de riesgo tromboembólico ${ }^{6}$.

Nuestra paciente se somete a dos revisiones quirúrgicas por sangrado donde no se evidencia foco hemorrágico susceptible de reparación quirúrgica, sino hematomas y sangrado en sábana, lo que sugiere que la coagulopatía podría estar implicada en este sangrado. Sin embargo, los análisis de coagulación convencionales eran rigurosamente normales (Tabla 1), lo que motivó que no se transfundiera fibrinógeno ni factores de coagulación hasta la realización de una prueba viscoelástica. En este contexto de sangrado agudo y grave puede producirse un déficit adquirido de FXIII?. Nuestra 
paciente no presentaba antecedentes personales ni familiares de eventos hemorrágicos previos y se confirmó posteriormente que, una vez resuelto el cuadro, la dosificación de FXIII estaba en rango normal al igual que en su seguimiento evolutivo durante el año siguiente. Podemos descartar que se trate de un déficit congénito de FXIII y afirmar que es un déficit adquirido.

El FXIII ancla la a2-antiplasmina al fibrinógeno, lo que asegura la estabilidad mecánica de la malla de fibrina y su resistencia fibrinolítica. Por lo tanto, un déficit de FXIII se asocia a la formación de un coágulo friable, susceptible de fibrinólisis precoz, como muestra la figura 1. El FXIII también está presente en las plaquetas, por lo que una plaquetopenia asociada, como en nuestro caso, disminuye aún más su disponibilidad ${ }^{8}$ y contribuye al sangrado. Además, las plaquetas contienen en sus gránulos grandes reservas de moléculas con capacidad antifibrinolítica ( $\alpha 2$-antiplasmina e inhibidor del activador del plasminógeno [PAl-1), por lo contribuyen a disminuir la fibrinólisis ante un daño tisular $^{9}$. Por tanto, su déficit agrava aún más esta situación de hiperfibrinólisis. El déficit adquirido de FXIII es frecuente en el perioperatorio y presenta una clínica mucho más pronunciada que el congénito, además responde a terapia de reemplazo, que es la reposición de este factor, ya sea con concentrado de factor purificado o con plasma fresco congelado.

El déficit de FXIII no se detecta en los análisis de coagulación convencional. Los MVE (tromboelastografía y tromboelastometría rotacional $)^{10}$, al realizar un análisis de la cinética global de formación y lisis del coágulo, son capaces de identificar de manera indirecta un déficit del FXIII. Tras la realización de la tromboelastometría consideramos que nuestra paciente presentaba hiperfibrinólisis franca: una línea plana en el FIBTEM es un signo descrito de hiperfibrinólisis ${ }^{11}$. Dicha hiperfibrinólisis es probablemente la responsable de obtener un análisis trombolestastómetrico tan alterado con una coagulación en rango normal, ya que los análisis de coagulación convencionales no detectan hiperfibrinólisis.

Llama la atención que en un primer momento no administramos FXIII, ya que tratamos a la paciente con complejos protrombínicos activados que aportan FII, FVII, FIX y FX. Sin embargo, el coágulo se normalizó según la tromboelastometría y, a nivel clínico, la paciente se estabilizó (se pudo retirar el soporte vasoactivo y dejó de exteriorizar sangrado). Pensamos que es debido al tratamiento de la hiperfibrinólisis con ácido tranexámico y al aporte de fibrinógeno a dosis altas. Hay evidencia a nivel científico de que dosis altas de fibrinógeno estabilizan la malla de fibrina, incluso en situaciones de déficit plaquetario ${ }^{12,13}$. La tercera tromboelastometría obtenida de la paciente (Fig. 3) es compatible con esta situación.

En nuestro caso pensamos que si se hubiera usado la tromboelastometría desde el inicio y se hubiera tratado a la paciente con ácido tranexámico y plasma a partir del segundo día postoperatorio podría haberse disminuido drásticamente la morbilidad y el riesgo de mortalidad (transfusiones de hemoderivados, dos reintervenciones y varias situaciones de shock hemorrágico). En nuestra unidad no disponemos de un protocolo ante sangrado masivo guiado por ROTEM. La utilización del mismo está ampliamente recomendada en este contexto $^{2,6}$, por lo que sería otra de las limitaciones observadas en el manejo de esta paciente.

Este caso enfatiza la utilidad de la tromboelastometría ante la sospecha clínica de hiperfibrinólisis, entidad no detectada por las analíticas de coagulación convencional. En nuestra opinión se debería utilizar un MVE de manera rutinaria en la cirugía con QIOH, ya que asocia riesgo de hiperfibrinólisis.

\section{Conflicto de intereses}

Los autores declaran no tener ningún conflicto de intereses.

\section{Financiación}

Los autores declaran no haber recibido financiación.

\section{Responsabilidades éticas}

Protección de personas y animales. Los autores declaran que para esta investigación no se han realizado experimentos en seres humanos ni en animales.

Confidencialidad de los datos. Los autores declaran que han seguido los protocolos de su centro de trabajo sobre la publicación de datos de pacientes.

Derecho a la privacidad y consentimiento informado. Los autores declaran que en este artículo no aparecen datos de pacientes.

\section{Bibliografía}

\footnotetext{
1. Pomel C, Ferron G, Lorimier G, Rey A, Lhomme C, Classe JM, et al. Hyperthermic intra-peritoneal chemotherapy using oxaliplatin as consolidation therapy for advanced epitelial ovarian caricinoma. Results of a II phase prospective multicentre trial. CHIPOVAC study. Eur J Surg Oncol J Eur Soc Surg Oncol Br Assoc Surg Oncol. 2010;36(6):589-93.

2. Kearsley R, Egan S, McCaul C. Anesthesia for cytoreductive surgery with hyperthermic intraperitoneal chemotherapy. Anesthesia Tutorial Of the Week. 2018;379:1-7
} 
Rev Argent Anestesiol. 2018;76

3. Raspe C, Flother L, Schneider R, Bucher M, Piso P. Best practice for perioperative management of patients with cytoreductive surgery and HIPEC. Eur J Surg Oncol. 2017;43(6):1013-27.

4. Hurdle H, Bishop G, Walker A, Moazeni A, Paloucci EO, Temple W, et al. Coagulation after cytoreductive surgery and hyperthermic intraperitoneal chemotherapy: a retrospective cohort analysis. Can $J$ Anaesth. 2017;64(11):1144-52.

5. Falcón Araña L, Fuentes-García D, Roca Calvo MJ, Hernández-Palazón J, Gil Martínez J, et al. Alterations in hemostasis during cytoreductive surgery and hyperthermic intraperitoneal chemotherapy in patients with peritoneal carcinomatosis. Cir Esp. 2015;93(8):496-501.

6. Sargant N, Roy A, Simpson S, Chandrakumaran K, Alves S, Coakes J, et al. A protocol for management of blood loss in surgical treatment of peritoneal malignancy by cytoreductive surgery and hyperthermic intraperitoneal chemotherapy. Transfusion Med. 2016;26(2):118-22

7. Korte W. FXIII in perioperative coagulation management. Best Pract Res Clin Anaesthesiol. 2010;24(1):85-93.

8. Von Rappard S, Hinnen C, Lussmann R, Rechsteiner M, Korte W. Factor $\mathrm{XIII}$ deficiency and trombocitopenia are frequent modulators of postope- rative clot firmness in a surgical intensive care unit. Transfus Med Hemother. 2017; 44(2):85-92

9. Moore HB, Moore EE, Gonzalez E, Hansen KC, Dzieciatkowska M, Chapman MP, et al. Hemolysis exacerbates hyperfibrinolysis, whereas platelolysis shuts down fibrinolysis: evolving concepts of the spectrum of fibrinolysis in response to severe injury. Shock. 2015;43(1):39-46.

10. Wikkelsø A, Wetterslev J, Møller AM, Afshari A. Thormboelastography (TEG) or rotational thromboelastometry (ROTEM) to monitor haemostatic treatment in bleeding patients: a systematic review with meta-analysis and trial sequential analysis. Anaesthesia. 2017;72(4):519-31.

11. Lier H, Vorweg M, Hanke A, Görlinger K. Thromboelastometry guided therapy of severe bleeding. Hamostaselogie. 2013;33(1):51-61.

12. Lang $\mathrm{T}$, Johanning $\mathrm{K}$, Metzler $\mathrm{H}$, Piepenbrock $\mathrm{S}$, Solomon $\mathrm{C}$, Rahe-Meyer $\mathrm{N}$, et al. The effects of fibrinogen levels on thromboelastometric variables in the presence of thrombocytopenia. Anesth Analg. 2009:108(3):751-8.

13. Kornblith LZ, Kutcher ME, Redick BJ, Calfee CS, Vilardi RF, Cohen MJ. Fibrinogen and platelet contributions to clot formation: implications for trauma resuscitation and thromboprophylaxis. J Trauma Acute Care Surg. 2014;76(2):255-6. 